\title{
Fast ion source and detector for investigating the interaction of turbulence with suprathermal ions in a low temperature toroidal plasma
}

\author{
G. Plyushchev, ${ }^{\text {a) }}$ A. Diallo, A. Fasoli, I. Furno, B. Labit, S. H. Müller, \\ M. Podestà, and F. M. Poli \\ Ecole Polytechnique Fédérale de Lausanne (EPFL), Centre de Recherches en Physique des Plasmas, \\ Association Euratom-Confédération Suisse, CH-1015, Lausanne, Switzerland \\ H. Boehmer, W. W. Heidbrink, and Y. Zhang \\ Department of Physics and Astronomy, University of California, Irvine, California 92697
}

(Received 5 May 2006; presented on 11 May 2006; accepted 28 May 2006; published online 21 September 2006)

\begin{abstract}
A specific experimental apparatus consisting of an ion source and a detector for the investigation of the interaction between suprathermal ions and drift-wave turbulence is developed on the toroidal plasma experiment. Due to the low plasma temperature $(\sim 5 \mathrm{eV})$, a spatially localized, small-size ion source $(\sim 4 \mathrm{~cm})$ mounted inside the vacuum vessel with relatively low ion energy $(\sim 100 \mathrm{eV}-1 \mathrm{keV})$ can be used. The source consists of an aluminosilicate Li-6 ion emitter $(6 \mathrm{~mm}$ diameter, 10-30 $\mu \mathrm{A}$ current) installed on a two-dimensional (2D) poloidally moving system. The location, energy, and current density profile of the ion beam will be measured using a 2D movable gridded energy analyzer. () 2006 American Institute of Physics. [DOI: 10.1063/1.2219407]
\end{abstract}

\section{INTRODUCTION}

The plasma of ITER and future thermonuclear reactors will be heated by the products of thermonuclear reactions (He-4) with very high energy $(\sim 3.5 \mathrm{MeV})$, in other words by fast nonthermal ions. These nonthermal ions will carry large amount of power ( $200 \mathrm{MW}$ for a fusion power of $1 \mathrm{GW})$. In existing tokamaks, fast ions can be generated by additional ion heating (neutral beam injection or ion cyclotron heating) ${ }^{1}$ or even electron heating. ${ }^{2}$ Highly energetic ions can exert a strong influence on plasma properties, such as temperature and density profiles, turbulence, equilibrium, etc., ${ }^{1}$ via direct heating, by exciting instabilities (Alfvén frequency modes) or by interacting with existing instabilities (e.g., sawteeth). Another very important question, related both to the basics physics of turbulence and to the anomalous transport, is how low-frequency plasma turbulence influences fast ions and, in turn, how fast ions can influence turbulence.

This problem can be addressed in the toroidal plasma experiment $^{3}$ (TORPEX) in a relatively simple experimental environment. Its plasma provides easy access for diagnostics, and is subject to drift-wave instabilities and turbulence. ${ }^{3}$ In this article we describe the main elements of the design of a fast ion source $(100 \mathrm{eV}-1 \mathrm{keV})$, a gridded energy analyzer, and a two-dimensional (2D) poloidally moving system for the source and the gridded energy analyzer to investigate the fast ion-turbulence interaction over almost the entire poloidal cross section.

\footnotetext{
${ }^{\text {a)} E l e c t r o n i c ~ m a i l: ~ g e n n a d y . p l y u s h c h e v @ e p f l . c h ~}$
}

\section{EXPERIMENTAL SETUP}

The TORPEX device has a major radius $R=1 \mathrm{~m}$, a minor radius $a=0.2 \mathrm{~m}$, a mainly toroidal magnetic field $B_{T}<0.1 \mathrm{~T}$, and a small vertical component $B_{z}<4 \mathrm{mT}$. The plasma is produced by microwaves launched from the low field side in the ordinary mode, with a frequency of $2.45 \mathrm{GHz}$ and a power $P_{\mathrm{rf}}<10 \mathrm{~kW}$. A toroidal electrical field $\left(E_{\text {loop }}<1.6 \mathrm{~V} / \mathrm{m}\right)$ can also be induced to produce a plasma current $\left(I_{p}<1 \mathrm{kA}\right.$ for $\left.\sim 3 \mathrm{~ms}\right)$ and to close magnetic flux surfaces in the plasma. The plasma discharge lasts for up to $1.8 \mathrm{~s}$. The typical electron temperature is $T_{e} \sim 5 \mathrm{eV}$ for both hydrogen and argon plasmas, with electron densities $n_{e} \sim 3 \times 10^{16} \mathrm{~m}^{-3}$ for hydrogen and $\sim 2 \times 10^{17} \mathrm{~m}^{-3}$ for argon (with addition of Ohmic power the density can rise up to $\sim 2 \times 10^{18} \mathrm{~m}^{-3}$ ). These low temperatures and densities allow highly localized measurements of plasma parameters with sets of different probes inserted directly in the plasma core. More than 200 channels are acquired simultaneously during the whole discharge, with a sampling rate of $250 \mathrm{kHz}$.

To investigate fast ion-turbulence interactions on TORPEX we should have a controllable fast ion source and diagnostics appropriate to measure the fast ion beam parameters, the background plasma profiles, and the turbulence properties. TORPEX plasmas are characterized by electrostatic fluctuations and turbulence originating from driftinterchange instabilities, driven by pressure gradient and magnetic curvature, i.e., mechanism relevant to magnetic fusion. ${ }^{4}$ The fast ion energy should be significantly higher than the plasma temperature, but low enough that fast ions are confined by the toroidal magnetic field. For TORPEX these conditions are satisfied for fast ion energies from $\sim 100 \mathrm{eV}$ to $\sim 1 \mathrm{keV}$ for Li-6 single ionized particles. The Li-6+ ion with $100 \mathrm{eV}$ energy has a Larmor radius of about 


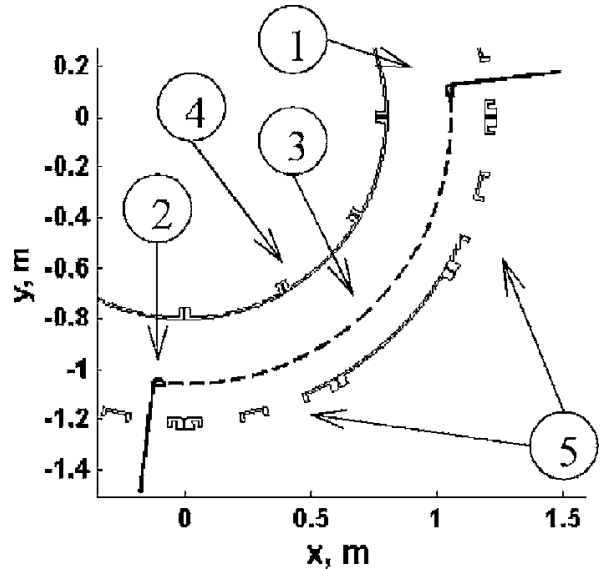

FIG. 1. Toroidal cross section of TORPEX with (1) fast ion source with support, (2) gridded energy analyzer, (3) trajectory of fast ions, (4) vacuum vessel, and (5) ports.

$3.5 \mathrm{~cm}$ (for $B_{T}=0.1 \mathrm{~T}$ ). This particle makes one toroidal turn in $0.11 \mathrm{~ms}$. The slowing down time for typical TORPEX plasma parameters due only to classical collisions is $\sim 30 \mathrm{~ms}$. As this is much shorter than the energy diffusion time $(\sim 7 \mathrm{~s})$, the ion beam is expected to remain monoenergetic. The critical energies for typical hydrogen and argon plasmas are equal to 440 and $180 \mathrm{eV}$, respectively; below these energies the collisions of the fast ions with thermal ions dominate over fast ion-electron collisions.

After injection, the beam tends to diverge due to at least six mechanisms: particle drifts, space charge, classical transport, poloidal electric field, turbulent transport, and interaction with Alfvénic modes. Experiments with and without plasma but with magnetic field will help us separate the first two from the last four mechanisms. Calculations based on the single particle approximation show the possibility to control the beam trajectory and the particle drift divergence with the vertical magnetic field. The space charge divergence is negligible because of the small beam current. The divergence due to poloidal electric field, turbulent transport, and interaction with Alfvénic modes will be investigated using a gridded energy analyzer. Both the source and the analyzer will be installed on 2D poloidally moving systems in two different cross sections (Fig. 1) to be able to change the fast ion current deposition and to measure the fast ion current profile.

The investigations of plasma turbulence and background profiles will be carried out with the help of Langmuir probes that already exist on TORPEX and some diagnostics which will be developed soon. These include a laser induced fluorescence (LIF) system for the ion velocity distribution function measurements, a fast camera (sampling rate up to $250 \mathrm{kHz}$ ) in the visible range for nonperturbative, direct fluctuation measurements, a set of edge Langmuir probes that will expand the coverage area of the existing probes, and a $2 \mathrm{D}$ poloidally movable flux probe.

\section{A. Fast ion source}

Due to the relatively low energy required, a small cylindrical ion source $(\sim 4 \mathrm{~cm}$ length, $2 \mathrm{~cm}$ diameter $)$ can be used. Such ion source can be installed directly inside the
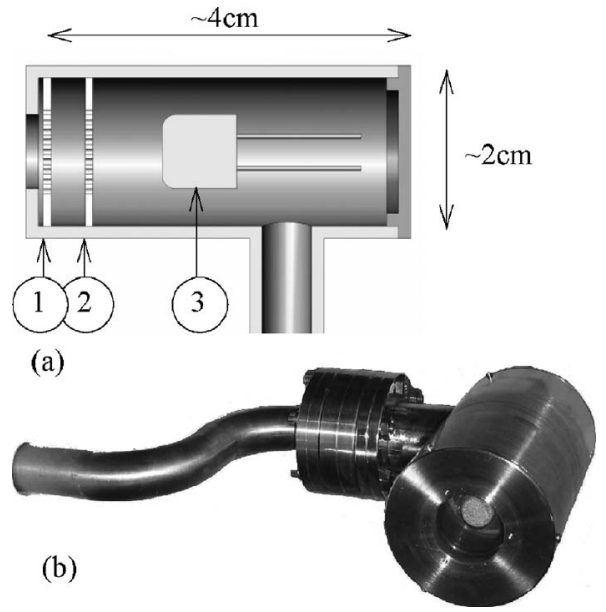

FIG. 2. (a) Scheme of the source. Shown are (1) screen grid, (2) accelerating grid, and (3) fast ion source. (b) Example of the Li ion source (characteristic dimension $\sim 10 \mathrm{~cm}$ ) which was developed in the University of California, Irvine.

vacuum vessel. The ion source consists of two aligned grids (screen grid and accelerating grid) and an ion emitter (Fig. 2). The screen grid will have the same potential as the plasma to minimize perturbations. The accelerating grid will be powered by a high voltage modulated power supply. The beam can be focused by varying the voltage between the accelerating and the screen grids. The wide energy range of fast ions $(100 \mathrm{eV}-1 \mathrm{keV}$, the upper limit being determined by dielectric breakdown voltage of the insulators) will allow us to investigate the energy dependencies. The use of light ions is preferable because such ions will have relatively high speed, which facilitates the fast ion-electron interactions. The current density of these ions should be small enough to prevent collective wave excitation and to avoid two stream instabilities. The emitter should cause low gas load to minimize the perturbation to the background plasma. To satisfy these requirements, an aluminosilicate $\mathrm{Li}-6+$ ion emitter has been chosen. ${ }^{5}$ The emitter has $6 \mathrm{~mm}$ beam diameter and 10-30 $\mu \mathrm{A}$ beam current, which depends on the extracting voltage according to the Schottky effect, and operates at a cathode temperature of about $1000{ }^{\circ} \mathrm{C}$. The lifetime of the emitter is expected to exceed $300 \mathrm{~h}$. Such ion source is not sensitive to magnetic fields up to $0.1 \mathrm{~T}$, which is appropriate for TORPEX.

\section{B. Gridded energy analyzer}

A gridded energy analyzer consisting of two grids and an anode will give information about the location, energy, and current density profile of the ion beam. The decelerating grid will be biased with a sweeping voltage (frequency $f \sim 1 \mathrm{kHz})$ that will determine a temporal resolution of at least $1 \mathrm{~ms}$. The spatial resolution is determined by the inlet diameter of the analyzer $(6 \mathrm{~mm})$ and by the accuracy of the 2D system positioning $(<5 \mathrm{~mm})$. An energy resolution of $1 \mathrm{~V}$ will be enough to measure fast ion beam energy profile in order to investigate transport both in real and velocity spaces which could be caused by nonclassical interactions with plasma. 


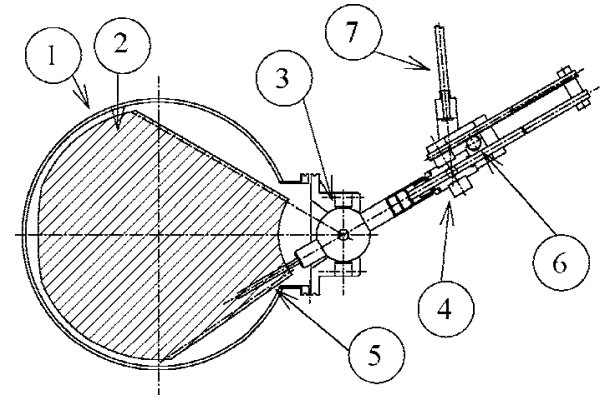

FIG. 3. Drawing of 2D poloidally moving system. The main elements of the drawing are (1) vacuum vessel, (2) coverage area, (3) angular and (4) linear motion feedthroughs, (5) ceramic tube, (6) remote linear motion system, and (7) arm for remote angular motion.

\section{2D poloidally moving system}

The fluctuation and turbulence level strongly depend on background plasma profiles (e.g., on the position on the pressure gradient), and for different regimes the region of interest may vary in the poloidal cross section. ${ }^{3}$ Thus, it is necessary to install both the fast ion source and the gridded energy analyzer on a $2 \mathrm{D}$ poloidally moving system to be able to change the fast ion deposition radially and vertically and to measure the fast ion current profile over the whole plasma cross section. Considering the high price and the limited lifetime of conventional bellows, for both motions we have chosen to use sliding seal feedthroughs with differential pump- ing (Fig. 3), a solution similar to that adopted on large plasma device (LAPD). ${ }^{6}$ The design of the angular feedthrough based on a steel ball allows an angular excursion of up to $70^{\circ}$. Ceramic tubing will support the probe inside the plasma column. As a consequence, the linear feedthrough will be placed approximately $40 \mathrm{~cm}$ away from the ball (see Fig. 3). Such construction gives us the possibility to cover almost the entire poloidal cross section, except for a small upper-right part, a limitation caused by outside structures of TORPEX. The positioning of the source and the detector will be achieved remotely using stepping motors and controlled through software.

\section{ACKNOWLEDGMENT}

This work is partly funded by the "Fonds National Suisse de la Recherche Scientifique."

${ }^{1}$ ITER Physics Base Editors, Nucl. Fusion 39, 2137 (1999).

${ }^{2}$ A. N. Karpushov, S. Coda, and B. P. Duval, Europhysics Conference Abstracts, Proceedings of the 30th EPS Conference on Controlled Fusion and Plasma Physics, St. Petersburg, Russia, 7-11 July 2003, edited by R. Koch and S. Lebedev (Plasma Physics Division of the European Physical Society (EPS), Mulhouse, 2003), Vol. 27A Pt. 3, p. 123.

${ }^{3}$ A. Fasoli, B. Labit, M. McGrath, S. H. Müller, M. Podestà, F. M. Poli, and G. Plyushchev, Bull. Am. Phys. Soc. 48, 119 (2003).

${ }^{4}$ F. M. Poli, B. Labit, M. McGrath, S. H. Müller, M. Podestà, F. M. Poli, and G. Plyushchev, Phys. Plasmas 13, 055902 (2006).

${ }^{5}$ http://www.cathode.com/pdf/tb-118.pdf

${ }^{6}$ D. Leneman and W. Gekelman, Rev. Sci. Instrum. 72, 3473 (2001). 